\title{
CURVATURE AND INTEGRABILITY OF ALMOST COMPLEX STRUCTURES
}

\author{
JIANMING WAN
}

\begin{abstract}
This note is concerned in so called harmonic complex structures introduced by the author previously. I will recall some previous results and emphasize the motivation: Provide an attempt to a fundamental problem in geometry-determining the complex structures on an almost complex manifold. I also discuss the almost-Hermitian case of harmonic complex structures and the connections with balanced structures.
\end{abstract}

\section{INTRODUCTION}

Let $M$ be an almost complex manifold. This means that there exists a smooth section $J \in \Gamma\left(T^{*} M \otimes T M\right)=\Gamma(\operatorname{hom}(T M, T M))$ satisfying

$$
J^{2}=-i d .
$$

The $J$ is called almost complex structure of $M$. An almost complex manifold must be oriented and has even dimension. Determining an almost complex structure on a manifold is a purely topological problem (equivalent to the structure groups of tangent bundle $G L(2 n, \mathbb{R})$ can reduce to $G L(n, \mathbb{C})$ ) and has been studied well [5]. In principle, we always can determine whether a given manifold has an almost complex structure (though the procedure may be very complex). In dimension 4 we have a fundamentally topological criterion of Wen-tsun Wu to determining the almost complex structure [7].

An almost complex structure $J$ on $M$ is said to be integrable, if it can induce a complex (manifold) structure. By the famous Newlander-Nirenberg theorem [4], $\mathrm{J}$ is integrable if and only if the Nijenhuis tensor vanishes, i.e.

$$
N(J)(X, Y)=J[J X, Y]+J[X, J Y]+[X, Y]-[J X, J Y] \equiv 0,
$$

for all $X, Y \in \Gamma(T M)$. A fundamental problem in geometry is to determine the complex structures on an almost complex manifold. The case of 2-dimension is classical. Every surface is a complex manifold, which is called Riemann surface. In dimension 4, by combining Wu's criterion with some results in algebraic geometry we can construct many compact almost complex manifolds without any complex structure (c.f. [1] page 167). For instance, $S^{1} \times S^{3} \sharp S^{1} \times S^{3} \sharp S^{2} \times S^{2}$ and $S^{1} \times S^{3} \sharp S^{1} \times S^{3} \sharp \mathbb{C} P^{2}$. However, up to now, we do not find a single higher dimensional manifold with almost complex structures but no complex structure. The higher dimensional examples seem to be existent undoubtedly. But another opinion of Yau (c.f. [11] problem 52) asserts that every compact almost complex manifold of dimension $\geq 6$ admits a complex structure. As well known, one can construct an almost complex structure on $S^{6}$ by using quaternions. But this almost complex structure is not integrable. It is an outstanding problem to determine the complex structures on $S^{6} . S^{6}$ is a touchstone to understand the complex structures of higher dimensional manifolds .

2010 Mathematics Subject Classification. Primary 53C15; Secondary 53C20.

Key words and phrases. curvature, almost complex structure.

The author is supported by National Natural Science Foundation of China No.11301416 and No.11601421. 
To deal with the fundamental problem, there are two folds: 1) To show the existence of complex structures, we should find some effective sufficient conditions to the existence of complex structures; 2) To show the nonexistence of complex structures, we need to find some obstructions. The sufficient conditions or obstructions should be involved in the geometry or topology of manifolds.

We are mainly concerned in the connections between complex structures and curvature of manifolds. Let $(M, J)$ be an almost complex manifold. We give a Riemannian metric $g$ on $M$. Then we can define the Hodge-Laplace operator $\Delta$ acting on tangent bundlevalued differential forms. Since $J$ can be seen as a tangent bundle-valued 1-forms, we may consider the action of $\Delta$ on $J$. When the manifold is compact, in [6] the author observed that

$$
\Delta J=0
$$

implies $J$ is integrable. On the other hand, the Bochner formula of $\Delta J$ contains curvature terms. So we can connect the integrability of almost complex structures with the geometry (curvature) of manifolds. In a sense this provides a probability for studying of existence of complex structures.

\section{BOCHNER TECHNIQUES FOR TANGENT BUNDLE-VALUED DIFFERENTIAL FORMS}

The materials in this section are standard, which can be found in many literatures. For example [8].

2.1. Hodge-Laplace operator. Notations. $\left\{e_{i}, 1 \leq i \leq n\right\}$ : local orthonormal frame field; $X, X_{0}, X_{1}, \cdots, X_{p}$ : smooth sections of tangent bundle $T M ; \omega, \theta$ : smooth sections of $\wedge^{p} T^{*} M \otimes$ $T M$.

Let $(M, g)$ be a Riemannian manifold. Let $\nabla$ be the Levi-Civita connection associated with $g . \nabla$ can be extended canonically to $\Gamma\left(\wedge^{p} T^{*} M \otimes T M\right)$ by

$$
\left(\nabla_{X} \omega\right)\left(X_{1}, \cdots, X_{p}\right)=\nabla_{X}\left(\omega\left(X_{1}, \cdots, X_{p}\right)\right)-\sum_{k=1}^{p} \omega\left(X_{1}, \cdots, \nabla_{X} X_{k}, \cdots, X_{p}\right) .
$$

We can define the differential operator $d: \Gamma\left(\wedge^{p} T^{*} M \otimes T M\right) \longrightarrow \Gamma\left(\wedge^{p+1} T^{*} M \otimes T M\right)$,

$$
d \omega\left(X_{0}, \cdots, X_{p}\right)=\sum_{k=0}^{p}(-1)^{k}\left(\nabla_{X_{k}} \omega\right)\left(X_{0}, \cdots, \hat{X}_{k}, \cdots, X_{p}\right),
$$

where $\hat{X}_{k}$ denotes removing $X_{k}$. The co-differential operator $\delta: \Gamma\left(\wedge^{p} T^{*} M \otimes T M\right) \longrightarrow$ $\Gamma\left(\wedge^{p-1} T^{*} M \otimes T M\right)$ is given by

$$
\delta \omega\left(X_{1}, \cdots, X_{p-1}\right)=-\sum_{i=1}^{n}\left(\nabla_{e_{i}} \omega\right)\left(e_{i}, X_{1}, \ldots, X_{p-1}\right) .
$$

The Hodge-Laplace operator is defined by

$$
\Delta \triangleq d \delta+\delta d \text {. }
$$

For any $\omega, \theta \in \Gamma\left(\wedge^{p} T^{*} M \otimes T M\right)$, we have the induced inner product

$$
\langle\omega, \theta\rangle \triangleq \sum_{1 \leq i_{1}<\cdots<i_{p} \leq n}\left\langle\omega\left(e_{i_{1}}, \cdots, e_{i_{p}}\right), \theta\left(e_{i_{1}}, \cdots, e_{i_{p}}\right)\right\rangle,
$$

If $M$ is compact, we have the global inner product

$$
(\omega, \theta) \triangleq \int_{M}\langle\omega, \theta\rangle d v
$$


By the self-adjoint property of $\Delta$, we have

$$
(\Delta \omega, \omega)=(d \omega, d \omega)+(\delta \omega, \delta \omega) \geq 0 .
$$

So $\Delta \omega=0$ if and only if $d \omega=0$ and $\delta \omega=0$.

We should mention that in general $d^{2} \neq 0$. For $A \in \Gamma\left(T^{*} M \otimes T M\right), d^{2} A\left(X_{1}, X_{2}, X_{3}\right)=$ $R\left(X_{3}, X_{2}\right) A X_{1}+R\left(X_{1}, X_{3}\right) A X_{2}+R\left(X_{2}, X_{1}\right) A X_{3}$.

\subsection{Weitzenböck formula.}

Proposition 2.1. For any tangent bundle-valued p-form $\omega$, we have

$$
\Delta \omega=-\nabla^{2} \omega+S
$$

where $\nabla^{2} \omega=\nabla_{e_{i}} \nabla_{e_{i}} \omega-\nabla_{\nabla_{e_{i} e_{i}}} \omega$ and $S\left(X_{1}, \cdots, X_{p}\right)=(-1)^{k}\left(R\left(e_{i}, X_{k}\right) \omega\right)\left(e_{i}, X_{1}, \cdots, \hat{X}_{k}, \cdots, X_{p}\right)$, for any $X_{1}, \cdots, X_{p} \in \Gamma(T M) . R$ is the curvature tensor $R(X, Y)=-\nabla_{X} \nabla_{Y}+\nabla_{Y} \nabla_{X}+\nabla_{[X, Y]}$ and $\left\{e_{i}\right\}$ is the local orthonormal frame field.

In fact the Bochner technique can work on any Riemannian vector bundles. For our purpose we only focus on tangent bundles.

\section{HaRmonic COMPLEX STRUCTURES}

From now on we assume that $(M, J)$ is a compact almost complex manifold. We give a Riemannian metric $g$ on $M$. $J$ does not need to be compatible with $g$. The author introduced the following concept in [6].

Definition 3.1. We say that $J$ is a harmonic complex structure if $\Delta J=0$.

By the self-adjoint property, $\Delta J=0$ if and only if $d J=0$ and $\delta J=0$. Recall that a Kähler structure means an almost complex structure $J$ compatible with $g$ and satisfying $\nabla J=0$. So a Kähler structure must be a harmonic complex structure. The following observation shows the meaning of harmonic complex structure.

Proposition 3.2. [6] A harmonic complex structure is integrable.

Proof. We only need to show that the Nijenhuis tensor 1.2 vanishes. By direct computation, one has

$$
\begin{aligned}
d J(X, Y)-d J(J X, J Y) & =\left(\left(\nabla_{X} J\right) Y-\left(\nabla_{Y} J\right) X\right)-\left(\left(\nabla_{J X} J\right) J Y-\left(\nabla_{J Y} J\right) J X\right) \\
& =[J X, Y]+[X, J Y]+J[J X, J Y]-J[X, Y] \\
& =-J N(J)(X, Y) .
\end{aligned}
$$

Since $\Delta J=0$ implies $d J=0$, hence $N(J)=0$.

Denote $K$ : Kähler structures; $H$ : harmonic complex structures; $C$ : complex structures. We have the inclusion relation

$$
K \subset H \subset C \text {. }
$$

Let $e(J)=\frac{1}{2}\left|J e_{i}\right|^{2}$ denote the energy density of $J$. Applying proposition 2.1 to $J$, we can obtain the Bochner type formula.

Theorem 3.3. [6]

$$
\Delta e(J)+\langle\Delta J, J\rangle=|\nabla J|^{2}+\left\langle J R\left(e_{i}, e_{j}\right) e_{i}, J e_{j}\right\rangle-\left\langle R\left(e_{i}, e_{j}\right) J e_{i}, J e_{j}\right\rangle,
$$

where $|\nabla J|^{2}=\left|\left(\nabla_{e_{i}} J\right)\left(e_{j}\right)\right|^{2}$. 
Proof. Following the notations in proposition 2.1, we can check that

$$
\langle S, J\rangle=\left\langle J R\left(e_{i}, e_{j}\right) e_{i}, J e_{j}\right\rangle-\left\langle R\left(e_{i}, e_{j}\right) J e_{i}, J e_{j}\right\rangle
$$

and

$$
\left\langle\nabla^{2} J, J\right\rangle=\Delta e(J)-|\nabla J|^{2} .
$$

Then the theorem is straightforward from formula 2.7

The below table gives the comparative relations.

TABLE 1.

\begin{tabular}{|c|c|}
\hline Kahler structure & harmonic complex structure \\
\hline totally geodesic map & harmonic map \\
\hline totally geodesic submanifold & minimal submanifold \\
\hline
\end{tabular}

4. SOME APPLICATIONS OF HARMONIC COMPLEX STRUCTURES

From theorem 3.3 , we have

$$
(d J, d J)+(\delta J, \delta J)=(\Delta J, J)=\int_{M}\left(|\nabla J|^{2}+\left\langle J R\left(e_{i}, e_{j}\right) e_{i}, J e_{j}\right\rangle-\left\langle R\left(e_{i}, e_{j}\right) J e_{i}, J e_{j}\right\rangle\right) d v \geq 0 .
$$

So $J$ is a harmonic complex structure if and only if

$$
\int_{M}\left(|\nabla J|^{2}+\left\langle J R\left(e_{i}, e_{j}\right) e_{i}, J e_{j}\right\rangle-\left\langle R\left(e_{i}, e_{j}\right) J e_{i}, J e_{j}\right\rangle\right) d v=0 .
$$

Combining proposition 3.2, we obtain a geometric sufficient condition of integrability of an almost complex structure.

Theorem 4.1. If

$$
\int_{M}\left(|\nabla J|^{2}+\left\langle J R\left(e_{i}, e_{j}\right) e_{i}, J e_{j}\right\rangle-\left\langle R\left(e_{i}, e_{j}\right) J e_{i}, J e_{j}\right\rangle\right) d v=0,
$$

then $J$ is integrable.

Though we do not know whether the 6-sphere $S^{6}$ has a complex structure, as an application of theorem 3.3 we have

Theorem 4.2. [6] $S^{6}$ with standard metric (or with small perturbation) can not admit any harmonic complex structure.

Proof. We only need to show that the right of formula 3.1 is positive. Under standard metric, the sectional curvature is equal to 1 . One can easily to show that $\left\langle J R\left(e_{i}, e_{j}\right) e_{i}, J e_{j}\right\rangle=$ $10 e(J) \geq 30$ and $\left\langle R\left(e_{i}, e_{j}\right) J e_{i}, J e_{j}\right\rangle=6$.

A well-known result of LeBrun [2] states that $S^{6}$ has no complex structure compatible with the standard metric. Kefeng Liu and Xiaokui Yang [9] [10] proved that $S^{6}$ can not admit a complex structure compatible with a metric such that the sectional curvature lies in $\left(\frac{1}{4}, 1\right]$. In our result the compatible condition is removed. But geometric restriction is increased.

We also can get a Kähler criterion for a harmonic complex structure. 
Theorem 4.3. [6] Let $J$ be an Hermitian harmonic complex structure. Then the scale curvature $S \leq\left\langle R\left(e_{i}, e_{j}\right) J e_{i}, J e_{j}\right\rangle$. The equal holds if and only if $J$ is a Kähler structure.

Proof. Since $J$ is an Hermitian harmonic complex structure, $e(J)=$ constant. The left of formula 3.1 equals to zero. So $S-\left\langle R\left(e_{i}, e_{j}\right) J e_{i}, J e_{j}\right\rangle=-|\nabla J|^{2} \leq 0$. The equal holds implies $\nabla J=0$. Namely $J$ is a Kähler structure.

\section{Almost-Hermitian case}

5.1. Almost-Hermitian manifold. We know that $\Delta J=0$ if and only if both $d J$ and $\delta J$ are equal to zero. Since we mainly are concerned in the integrability of almost complex structures, only the $d J=0$ is useful for our purpose. We should remove the condition $\delta J=0$. When $J$ is compatible with the Riemannian metric, i.e. $M$ is an almost-Hermitian manifold, we can do it. Our main observation is

Proposition 5.1. Let $M$ be an almost-Hermitian manifold. Then $d J=0$ implies $\delta J=0$. More precisely, for any $X \in \Gamma(T M)$ we have

i) $\langle J X, \delta J\rangle+\left\langle d J\left(X, e_{i}\right), J e_{i}\right\rangle \equiv 0$,

ii) $\langle X, \delta J\rangle+\left\langle d J\left(X, e_{i}\right), e_{i}\right\rangle \equiv 0$.

Proof. We choose the normal frame field (i.e. $\left.\nabla_{e_{i}} e_{j}\right|_{p}=0$ for a fixed point $p$ ). Then $d J\left(X, e_{i}\right)=\nabla_{X} J e_{i}-\nabla_{e_{i}} J X+J \nabla_{e_{i}} X$. Hence

$$
\begin{aligned}
\left\langle d J\left(X, e_{i}\right), J e_{i}\right\rangle & =\left\langle\nabla_{X} J e_{i}, J e_{i}\right\rangle-\left\langle\nabla_{e_{i}} J X, J e_{i}\right\rangle+\left\langle J \nabla_{e_{i}} X, J e_{i}\right\rangle \\
& =-\left\langle\nabla_{e_{i}} J X, J e_{i}\right\rangle+\left\langle\nabla_{e_{i}} X, e_{i}\right\rangle \\
& =\left\langle J X, \nabla_{e_{i}} J e_{i}\right\rangle-e_{i}\left\langle X, e_{i}\right\rangle+\operatorname{div} X \\
& =-\langle J X, \delta J\rangle-\operatorname{div} X+\operatorname{div} X=-\langle J X, \delta J\rangle
\end{aligned}
$$

and

$$
\begin{aligned}
\left\langle d J\left(X, e_{i}\right), e_{i}\right\rangle & =\left\langle\nabla_{X} J e_{i}, e_{i}\right\rangle-\left\langle\nabla_{e_{i}} J X, e_{i}\right\rangle+\left\langle J \nabla_{e_{i}} X, e_{i}\right\rangle \\
& =-\left\langle\nabla_{e_{i}} J X, e_{i}\right\rangle-\left\langle\nabla_{e_{i}} X, J e_{i}\right\rangle \\
& =-\operatorname{div} J X+\left\langle X, \nabla_{e_{i}} J e_{i}\right\rangle-e_{i}\left\langle X, J e_{i}\right\rangle \\
& =-\operatorname{div} J X-\langle X, \delta J\rangle+e_{i}\left\langle J X, e_{i}\right\rangle=-\langle X, \delta J\rangle .
\end{aligned}
$$

Now we explain why $d J=0$ can imply $\delta J=0$. Let $J_{t},-\epsilon<t<\epsilon$ be a family of almost complex structures. $J_{0}$ is compatible with the Riemannian metric. Then the energy density $e\left(J_{0}\right)=\frac{1}{2} \sum_{i=1}^{2 n}\left|J_{0} e_{i}\right|^{2}=n$. And

$$
\begin{aligned}
e\left(J_{t}\right)=\frac{1}{2} \sum_{i}\left|J_{t} e_{i}\right|^{2} & =\frac{1}{2} \sum_{i, j}\left(J_{i}^{j}\right)^{2}=\frac{1}{4} \sum_{i, j}\left(J_{i}^{j}\right)^{2}+\left(J_{j}^{i}\right)^{2}=\frac{1}{4} \sum_{i} \sum_{j}\left(J_{i}^{j}\right)^{2}+\left(J_{j}^{i}\right)^{2} \\
& \geq \frac{1}{2} \sum_{i}\left|\sum_{j} J_{i}^{j} J_{j}^{i}\right|=\frac{1}{2} \sum_{i} 1=n=e\left(J_{0}\right),
\end{aligned}
$$

where $J_{t} e_{i}=J_{i}^{j} e_{j}$. So the energy $E\left(J_{0}\right)=\int_{M} e\left(J_{0}\right) d v$ is minimal. Let us compare with harmonic maps. Let $f$ be a smooth map between two Riemannian manifolds $M$ and $N$. If the energy $E(f)=\int_{M} e(f) d v$ is minimal, then $f$ is a harmonic map and the trace $\delta(d f)=0$ [8]. So intuitively we should has $\delta J=0$.

Combining theorem 3.3 and proposition 5.1 we immediately have 
Theorem 5.2. Let $M$ be an almost-Hermitian manifold. Then $d J=0$ if and only if

$$
\int_{M}\left(|\nabla J|^{2}+S-\left\langle R\left(e_{i}, e_{j}\right) J e_{i}, J e_{j}\right\rangle\right) d v=0
$$

where $S$ denotes the scale curvature.

We use proposition 5.1 to give a vanishing result related to Nijenhuis tensor. This may be known elsewhere.

Theorem 5.3. Let $M$ be an almost-Hermitian manifold. Then for any $X \in \Gamma(T M)$ we have $\left\langle N(J)\left(X, e_{i}\right), e_{i}\right\rangle \equiv 0$.

Proof. By proposition 5.1.

$$
\begin{gathered}
\left\langle N(J)\left(X, e_{i}\right), e_{i}\right\rangle=\left\langle J N(J)\left(X, e_{i}\right), J e_{i}\right\rangle=\left\langle d J\left(J X, J e_{i}\right)-d J\left(X, e_{i}\right), J e_{i}\right\rangle \\
=\left\langle d J\left(J X, J e_{i}\right), J e_{i}\right\rangle-\left\langle d J\left(X, e_{i}\right), J e_{i}\right\rangle=-\langle J X, \delta J\rangle+\langle J X, \delta J\rangle=0 .
\end{gathered}
$$

5.2. Connections with the balanced metrics. Let $M$ be an almost-Hermitian manifold. Let $\omega$ given by $\omega(X, Y)=\langle X, J Y\rangle$ be the almost-Hermitian form. When $J$ is integrable, we say that $J$ induces a Kähler structure if $d \omega=0$ and a balanced structure if $d \omega^{m-1}=0$ $\left(m=\frac{\operatorname{dim} M}{2}\right)$ (c.f. [3]). We will show that

Theorem 5.4. Let $M$ be a compact almost-Hermitian $n$-manifold $(n=2 m)$. If formula 5.1 holds, then $J$ induces a balanced structure.

For a special case $n=4$, formula 5.1 implies that $J$ induces a Kähler structure.

Since formula 5.1 implies that $d J=0$ (hence $J$ is integrable) and $\delta J=0$. Then theorem 5.4 follows from below lemma.

Lemma 5.5. $d \omega^{m-1}=0$ if and only if $\delta J=0$.

Proof. We use same notation $\delta$ denote the co-differential operator in Hodge theory. Then $d \omega^{m-1}=0$ if and only if $\delta \omega=0$. For any $X \in \Gamma(T M)$,

$$
\begin{aligned}
-\delta \omega(X) & =i\left(e_{j}\right) \nabla_{e_{j}} \omega(X)=\left(\nabla_{e_{j}} \omega\right)\left(e_{j}, X\right) \\
& =\nabla_{e_{j}} \omega\left(e_{j}, X\right)-\omega\left(\nabla_{e_{j}} e_{j}, X\right)-\omega\left(e_{j}, \nabla_{e_{j}} X\right) \\
& =e_{j}\left\langle e_{j}, J X\right\rangle-\left\langle\nabla_{e_{j}} e_{j}, J X\right\rangle+\left\langle J e_{j}, \nabla_{e_{j}} X\right\rangle \\
& =\operatorname{div}(J X)+e_{j}\left\langle J e_{j}, X\right\rangle-\left\langle\nabla_{e_{j}} J e_{j}, X\right\rangle \\
& =\operatorname{div}(J X)-e_{j}\left\langle e_{j}, J X\right\rangle+\langle\delta J, X\rangle \\
& =\langle\delta J, X\rangle .
\end{aligned}
$$

For a Kähler structure we have the well-known relation between curvature tensor and complex structure: $R(X, Y) J Z=J R(X, Y) Z$. For a balanced manifold, we have that

Theorem 5.6. Let $M$ be a balanced manifold. Then $\left|S-\left\langle R\left(e_{i}, e_{j}\right) J e_{i}, J e_{j}\right\rangle\right| \leq|\nabla J|^{2}$. 
Proof. We choose a normal frame $\left\{e_{i}\right\}$. Then $|\nabla J|^{2}=\sum_{i, j}\left|\nabla_{e_{i}} J e_{j}\right|^{2},|d J|^{2}=\sum_{i<j} \mid \nabla_{e_{i}} J e_{j}-$ $\left.\nabla_{e_{j}} J e_{i}\right|^{2}$. Then

$$
\begin{aligned}
|d J|^{2} & =\sum_{i<j}\left(\left|\nabla_{e_{i}} J e_{j}\right|^{2}+\left|\nabla_{e_{j}} J e_{i}\right|^{2}-2\left\langle\nabla_{e_{i}} J e_{j}, \nabla_{e_{j}} J e_{i}\right\rangle\right) \\
& \leq 2 \sum_{i<j}\left(\left|\nabla_{e_{i}} J e_{j}\right|^{2}+\left|\nabla_{e_{j}} J e_{i}\right|^{2}\right) \\
& =2\left(\sum_{i, j}\left|\nabla_{e_{i}} J e_{j}\right|^{2}-\sum_{k}\left|\nabla_{e_{k}} J e_{k}\right|^{2}\right) \\
& \leq 2\left(|\nabla J|^{2}-\frac{|\delta J|^{2}}{n}\right) .
\end{aligned}
$$

When $\delta J=0$, by the formula 3.1 we can calculate directly that $|d J|^{2}=|\nabla J|^{2}+S-$ $\left\langle R\left(e_{i}, e_{j}\right) J e_{i}, J e_{j}\right\rangle$. So one has $-|\nabla J|^{2} \leq S-\left\langle R\left(e_{i}, e_{j}\right) J e_{i}, J e_{j}\right\rangle \leq|\nabla J|^{2}$.

Remark 5.7. Let $M$ be an almost complex $n$-manifold. The $J$ needs not be compatible with the Riemannian metric. Then

$$
-\int_{M}|\nabla J|^{2} d v \leq \int_{M}\left(\left\langle J R\left(e_{i}, e_{j}\right) e_{i}, J e_{j}\right\rangle-\left\langle R\left(e_{i}, e_{j}\right) J e_{i}, J e_{j}\right\rangle\right) d v \leq(n-1) \int_{M}|\nabla J|^{2} d v .
$$

We leave the proof of remark to the readers.

\section{Some REMARKs}

Our main purpose is to find some geometric obstructions or sufficient conditions to existence of complex structures. The obstructive problem is try to find a necessary and sufficient condition for Nijenhuis tensor vanishing. Namely

$$
N(J)=0 \Longleftrightarrow C(J)=0 .
$$

Here $C(J)$ is a global curvature expression related to $J$, which is similar to formula 4.1 or 5.1. Once we find such a relation, if a suitable geometric condition can leads to $C(J)>0(<$ 0 ) for any $J$, we can claim that the manifold does not admit a complex structure. Theorem 4.1 or 5.2 only gives a sufficient condition for Nijenhuis tensor vanishing.

To find an effective sufficient condition, professor Kefeng Liu suggests that we should use 3.1 to construct a suitable flow of almost complex structures. Under some suitable geometric condition the flow converges to an integrable one. In this case we must deal with the problem of how the evolution equation keeps the almost complex structures.

\section{REFERENCES}

1. W. Barth, K. Hulek, C. Peters and A. Van de Ven, Compact complex surfaces. Second edition. Ergebnisse der Mathematik und ihrer Grenzgebiete. 3. Folge. A Series of Modern Surveys in Mathematics [Results in Mathematics and Related Areas. 3rd Series. A Series of Modern Surveys in Mathematics], 4. Springer-Verlag, Berlin, 2004. xii+436 pp.

2. C. LeBrun, Orthogonal complex structure on $S^{6}$. Proc. Amer. Math. Soc., 1987, 101, 136-138.

3. M. L., Michelsohn, On the existence of special metrics in complex geometry. Acta Math. 149 (1982), no. 3-4, 261-295.

4. A. Newlander, A. and L. Nirenberg, Complex analytic coordinates in almost complex manifolds. Ann. of Math. (2) 65 (1957), 391-404.

5. N. Steenrod, The Topology of Fibre Bundles. Princeton Mathematical Series, vol. 14. Princeton University Press, Princeton, N. J., 1951. viii+224 pp.

6. Jianming, Wan, Harmonic complex structures. (Chinese) Chinese Ann. Math. Ser. A 30 (2009), no. 6, 761764. arXiv:1007.4392v1 [math.DG] 
7. Wen-Tsun, Wu Sur la structure presque complexe d'une variété différentiable réelle de dimension 4. (French) C. R. Acad. Sci. Paris 227, (1948). 1076-1078.

8. Yuanlong, Xin, Geometry of harmonic maps. Progress in Nonlinear Differential Equations and their Applications, 23. Birkhäuser Boston, Inc., Boston, MA, 1996.

9. Kefeng, Liu and Xiaokui, Yang Geometry of Hermitian manifolds. Internat. J. Math. 23 (2012) 40pp.

10. Kefeng, Liu and Xiaokui, Yang Ricci curvatures on Hermitian manifolds. arXiv:1404.2481.

11. S.T. Yau, Open problems in geometry. Differential geometry: partial differential equations on manifolds (Los Angeles, CA, 1990), 1-28, Proc. Sympos. Pure Math., 54, Part 1, Amer. Math. Soc., Providence, RI, 1993.

School of Mathematics, Northwest University, Xi'an 710127, China

E-mail address: wanj_m@aliyun.com 